\title{
COUNTING PERMUTATIONS BY NUMBERS OF EXCEDANCES, FIXED POINTS AND CYCLES
}

\author{
SHI-MEI MA
}

\author{
(Received 9 June 2011)
}

Abstract

In this paper we present a combinatorial proof of an identity involving the two kinds of Stirling numbers and the numbers of permutations with prescribed numbers of excedances and cycles. Several recurrence relations related to the numbers of excedances, fixed points and cycles are also obtained.

2010 Mathematics subject classification: primary 05A15; secondary 05A19.

Keywords and phrases: excedances, fixed points, cycles, permutations.

\section{Introduction}

Let $\mathcal{S}_{n}$ be the symmetric group on the set $[n]=\{1,2, \ldots, n\}$ and $\pi=\pi(1) \pi(2) \ldots$ $\pi(n) \in \mathcal{S}_{n}$. The number of excedances of $\pi$ is $\operatorname{exc}(\pi):=|\{1 \leq i \leq n: \pi(i)>i\}|$ and that of fixed points is $\operatorname{fix}(\pi):=|\{1 \leq i \leq n: \pi(i)=i\}|$. Denote by $\operatorname{cyc}(\pi)$ the number of cycles of $\pi$. Clearly, we have $\operatorname{exc}(\pi) \leq n-\operatorname{cyc}(\pi)$ for $\pi \in \mathcal{S}_{n}$. For example, the permutation $\pi=315426$ has the standard cycle decomposition $(1,3,5,2)(4)(6)$, so $\operatorname{exc}(\pi)=2$, $\operatorname{fix}(\pi)=2$ and $\operatorname{cyc}(\pi)=3$. We say that $\pi$ is a derangement if $\operatorname{fix}(\pi)=0$. Let $D_{n}$ be the set of all derangements in $\mathcal{S}_{n}$. The Eulerian polynomials $A_{n}(x)$ are defined by

$$
A_{0}(x)=1, \quad A_{n}(x)=\sum_{\pi \in \mathcal{S}_{n}} x^{\operatorname{exc}(\pi)+1} \quad \text { for } n \geq 1 .
$$

It is well known that the Eulerian polynomials $A_{n}(x)$ satisfy

$$
\sum_{k \geq 0} k^{n} x^{k}=\frac{A_{n}(x)}{(1-x)^{n+1}},
$$

and one representative form of $A_{n}(x)$ is the Frobenius formula

$$
A_{n}(x)=x \sum_{k=0}^{n} k ! S(n, k)(x-1)^{n-k} \quad \text { for } n \geq 1,
$$

This work was supported by the Fundamental Research Funds for the Central Universities (N100323013). (C) 2011 Australian Mathematical Publishing Association Inc. 0004-9727/2011 \$16.00 
where $S(n, k)$ are the Stirling numbers of the second kind. The numbers $S(n, k)$ count partitions of $[n]$ with $k$ disjoint, nonempty subsets (blocks, for short). We follow Comtet [4] for the basic definitions, notation and terminology.

In recent years, several authors have devoted attention to the statistics of excedances, fixed points and cycles (see [10-12], for instance). In [3], Brenti studied the $q$-Eulerian polynomials

$$
A_{0}(x, q)=1, \quad A_{n}(x, q)=\sum_{\pi \in \mathcal{S}_{n}} x^{\operatorname{exc}(\pi)} q^{\operatorname{cyc}(\pi)},
$$

and established the link with $q$-symmetric functions arising from plethysm. He obtained the recurrence relation

$$
A_{n+1}(x, q)=(n x+q) A_{n}(x, q)+x(1-x) \frac{d}{d x} A_{n}(x, q)
$$

[3, Proposition 7.2] and showed that $A_{n}(x, q)$ has only real simple zeros when $q$ is a positive rational number [3, Theorem 7.5]. Brenti [3, Conjecture 8.8] also conjectured that $A_{n}(x,-t)$ has only real zeros, where $n, t \in \mathbb{N}$. This conjecture has been settled by Brändén [2]. Moreover, by the theory of symmetric functions, Brenti [3, Proposition 7.3] proved that

$$
\sum_{n \geq 0} A_{n}(x, q) \frac{t^{n}}{n !}=\left(\frac{e^{t(x-1)}-x}{1-x}\right)^{-q}
$$

Let

$$
P_{0}(x, y, q)=1, \quad P_{n}(x, y, q)=\sum_{\pi \in \mathcal{S}_{n}} x^{\mathrm{exc}(\pi)} y^{\mathrm{fix}(\pi)} q^{\mathrm{cyc}(\pi)} \quad \text { for } n \geq 1 .
$$

Clearly, $A_{n}(x)=x A_{n}(x, 1)=x P_{n}(x, 1,1)$ and $A_{n}(x, q)=P_{n}(x, 1, q)$ for $n \geq 1$. Since

$$
\begin{aligned}
P_{n}(x, y, q) & =\sum_{i=0}^{n}\left(\begin{array}{l}
n \\
i
\end{array}\right)(y q-q)^{i} \sum_{\pi \in \mathcal{S}_{n-i}} x^{\operatorname{exc}(\pi)} q^{\operatorname{cyc}(\pi)} \\
& =\sum_{i=0}^{n}\left(\begin{array}{l}
n \\
i
\end{array}\right)(y q-q)^{i} A_{n-i}(x, q),
\end{aligned}
$$

it follows from (1.1) that

$$
\sum_{n \geq 0} P_{n}(x, y, q) \frac{t^{n}}{n !}=\left(\frac{e^{t(x-y)}-x e^{t(1-y)}}{1-x}\right)^{-q} .
$$

The case $q=1$ of (1.2) was given by Foata and Schützenberger [5, Theorem 4.2]. Using the exponential formula [14, Corollary 5.5.5], Ksavrelof and Zeng [7, p. 2] also obtained (1.2). In [9, Theorem 1], the author obtained the identity

$$
\sum_{i \geq 0} i^{n}\left(\begin{array}{c}
q+i-1 \\
i
\end{array}\right) x^{i}=\frac{x^{n}}{(1-x)^{n+q}} A_{n}\left(x^{-1}, q\right) \text {. }
$$

Combining (1.2) and (1.3), we immediately obtain the following result. 
Proposition 1.1. For $n \geq 0$,

$$
\sum_{k \geq 0}\left(\begin{array}{c}
q+k-1 \\
k
\end{array}\right)\left(k+\frac{y-1}{1-x} x q\right)^{n} x^{k}=\frac{x^{n}}{(1-x)^{n+q}} P_{n}\left(x^{-1}, y, q\right) .
$$

As a generalization of the classical Eulerian polynomials $A_{n}(x)$, if a polynomial $g(x)$ satisfies

$$
\sum_{k \geq 0} f(k) x^{k}=\frac{g(x)}{(1-x)^{d+1}},
$$

where $\operatorname{deg} g(x)=d$, then $g(x)$ is called the $f$-Eulerian polynomial [13, p. 209]. It is well known that $A_{n}(x)=x^{n+1} A\left(x^{-1}\right)$ for $n \geq 1$. It follows from Proposition 1.1 that the polynomial $P_{n}(x, y, q)$ may be seen as a generalized $f$-Eulerian polynomial.

Set

$$
a(n, k, i)=\left|\left\{\pi \in \mathcal{S}_{n}: \operatorname{exc}(\pi)=n-k, \operatorname{cyc}(\pi)=i\right\}\right|
$$

and

$$
p(n, s, t, r)=\left|\left\{\pi \in \mathcal{S}_{n}: \operatorname{exc}(\pi)=n-s, \operatorname{fix}(\pi)=t, \operatorname{cyc}(\pi)=r\right\}\right| .
$$

This paper is organized as follows. In the next section we combinatorially prove an identity involving the two kinds of Stirling numbers and the numbers $a(n, k, i)$. In Section 3 we present several recurrence relations involving the numbers $p(n, s, t, r)$.

\section{An identity}

There is an identity relating Stirling numbers of the second kind to Eulerian numbers, that is,

$$
r ! S(n, r)=\sum_{k=0}^{r} A(n, k)\left(\begin{array}{l}
n-k \\
r-k
\end{array}\right)
$$

(see [1, Theorem 1.17], for instance). Various $q$-generalizations of the identity (2.1) have been pursued by several authors (see [6], for instance). Recall that the signless Stirling number of the first kind $c(r, i)$ counts permutations of $[r]$ with exactly $i$ cycles, so $\sum_{i=1}^{r} c(r, i)=r$ !. As a refinement of (2.1), we obtain the following result.

THEOREM 2.1. For all nonnegative integers $n \geq r \geq i$,

$$
S(n, r) c(r, i)=\sum_{k=i}^{r} a(n, k, i)\left(\begin{array}{l}
n-k \\
r-k
\end{array}\right) .
$$

Proof. The left-hand side of (2.2) counts the following objects: first partition $[n]$ into $r$ blocks and then arrange the $r$ blocks into $i$ cycles. We will show that the right-hand side of (2.2) counts the same objects. For convenience, we will write $\pi \in \mathcal{S}_{n}$ in standard cycle decomposition, where each cycle is written with its smallest entry first and the cycles are written in increasing order of their smallest entry. Take a permutation

$$
\pi=\left(\pi\left(p_{1}\right), \ldots\right)\left(\pi\left(p_{2}\right), \ldots\right) \cdots\left(\pi\left(p_{i}\right), \ldots\right)
$$


counted by $a(n, k, i)$. If $i=r$, let us write down $r-1$ bars between the cycles of $\pi$, that is,

$$
\left(\pi\left(p_{1}\right), \ldots\right)\left|\left(\pi\left(p_{2}\right), \ldots\right)\right| \cdots \mid\left(\pi\left(p_{i}\right), \ldots\right) .
$$

Erasing the parentheses, we get a partition of $[n]$ with $r$ blocks. Hence $a(n, r, r)=$ $S(n, r)$.

If $i<r$, we will split some of the cycles of $\pi$. Now consider positions where $\pi(j)>j$. There are by definition $n-k$ such positions, choose $r-k$ of these positions to put a bar behind. Note that there are $\left(\begin{array}{l}n-k \\ r-k\end{array}\right)$ ways to choose these positions. Erasing the parentheses, we will get a partition of $[n]$ with $r$ blocks. It is easy to verify that each such partition will be obtained exactly $c(r, i)$ times. This completes the proof.

Recall a pair of inverse relations used in [1, p. 13].

LEMma 2.2. We have

$$
f(n, r)=\sum_{k=0}^{r} g(n, k)\left(\begin{array}{l}
n-k \\
r-k
\end{array}\right) \text { if and only if } g(n, k)=\sum_{r=0}^{k}(-1)^{k-r} f(n, r)\left(\begin{array}{l}
n-r \\
k-r
\end{array}\right) \text {. }
$$

Combining Theorem 2.1 and Lemma 2.2, we obtain an explicit formula for the numbers $a(n, k, i)$.

Corollary 2.3. For all nonnegative integers $n \geq k \geq i$,

$$
a(n, k, i)=\sum_{r=i}^{k}(-1)^{k-r} S(n, r) c(r, i)\left(\begin{array}{l}
n-r \\
k-r
\end{array}\right) \text {. }
$$

\section{Basic recurrence relations}

Let $A_{1}, A_{2}, \ldots, A_{n}$ be subsets of a finite set. We say that the sets $A_{1}, A_{2}, \ldots, A_{n}$ are exchangeable [4, p. 179] if and only if the cardinality of any intersection of $k$ arbitrary subsets among them depends only on $k$, where $1 \leq k \leq n$.

THEOREM 3.1. We have

$$
p(n, s, t, r)=\left(\begin{array}{c}
n \\
t
\end{array}\right) \sum_{j=0}^{n-t}\left(\begin{array}{c}
n-t \\
j
\end{array}\right)(-1)^{j} a(n-t-j, s-t-j, r-t-j) .
$$

In particular,

$$
p(n, s, 0, r)=\sum_{j=0}^{n}\left(\begin{array}{l}
n \\
j
\end{array}\right)(-1)^{j} a(n-j, s-j, r-j) .
$$

Proof. Let

$$
A_{i}=\left\{\pi \in \mathcal{S}_{n}: \operatorname{exc}(\pi)=n-s, \pi(i)=i, \operatorname{cyc}(\pi)=r\right\} \quad \text { for } 1 \leq i \leq n .
$$

Note that

$$
\left|A_{i_{1}} \cap A_{i_{2}} \cap \cdots \cap A_{i_{t}}\right|=a(n-t, s-t, r-t) \quad \text { for } 1 \leq t \leq n,
$$


which implies the exchangeability of the sets $A_{1}, A_{2}, \ldots, A_{n}$. Using the inclusionexclusion principle [4, p. 195, Theorem A], we obtain

$$
\begin{aligned}
p(n, s, t, r) & =\sum_{i=t}^{n}(-1)^{i-t}\left(\begin{array}{l}
i \\
t
\end{array}\right)\left(\begin{array}{l}
n \\
i
\end{array}\right) a(n-i, s-i, r-i) \\
& =\left(\begin{array}{l}
n \\
t
\end{array}\right) \sum_{j=0}^{n-t}(-1)^{j}\left(\begin{array}{c}
n-t \\
j
\end{array}\right) a(n-t-j, s-t-j, r-t-j) .
\end{aligned}
$$

The theorem is thus proved.

We now present a recurrence relation for the numbers $p(n, s, t, r)$.

Theorem 3.2. We have

$$
\begin{aligned}
p(n+1, s, t, r)=( & +1) p(n, s, t+1, r)+(s-t) p(n, s, t, r) \\
& +(n-s+1) p(n, s-1, t, r)+p(n, s-1, t-1, r-1) .
\end{aligned}
$$

Proof. We will write $\pi \in \mathcal{S}_{n}$ in standard cycle decomposition. Let $n$ be a fixed positive integer. Let $\sigma_{i} \in S_{n+1}$ be the permutation obtained from $\sigma \in \mathcal{S}_{n}$ by inserting the entry $n+1$ just before $\sigma(i)$ if $i \in[n]$ or as a new cycle $(n+1)$ if $i=n+1$. Then

$$
\begin{gathered}
\operatorname{exc}\left(\sigma_{i}\right)= \begin{cases}\operatorname{exc}(\sigma) & \text { if } i \in[n] \text { and } \sigma(i)>i, \\
\operatorname{exc}(\sigma)+1 & \text { if } i \in[n] \text { and } \sigma(i) \leq i, \\
\operatorname{exc}(\sigma) & \text { if } i=n+1 ;\end{cases} \\
\operatorname{fix}\left(\sigma_{i}\right)= \begin{cases}\operatorname{fix}(\sigma)-1 & \text { if } i \in[n] \text { and } \sigma(i)=i, \\
\operatorname{fix}(\sigma) & \text { if } i \in[n] \text { and } \sigma(i) \neq i, \\
\operatorname{fix}(\sigma)+1 & \text { if } i=n+1 ;\end{cases}
\end{gathered}
$$

and

Recall that

$$
\operatorname{cyc}\left(\sigma_{i}\right)= \begin{cases}\operatorname{cyc}(\sigma) & \text { if } i \in[n] \\ \operatorname{cyc}(\sigma)+1 & \text { if } i=n+1\end{cases}
$$

$$
p(n+1, s, t, r)=\left|\left\{\sigma_{i} \in S_{n+1}: \operatorname{exc}\left(\sigma_{i}\right)=n+1-s, \operatorname{fix}\left(\sigma_{i}\right)=t, \operatorname{cyc}\left(\sigma_{i}\right)=r\right\}\right| .
$$

The following statements then hold.

(a) If $\sigma$ has $n-s$ excedances, $t+1$ fixed points and $r$ cycles, then we can insert the entry $n+1$ just before one of the $t+1$ fixed points of $\sigma$. We have $p(n, s, t+1, r)$ choices for $\sigma$. This case applies to the first term in the recurrence relation.

(b) If $\sigma$ has $n-s$ excedances, $t$ fixed points and $r$ cycles, then we can insert the entry $n+1$ just before one of the $s-t$ entries $\sigma(i)$ that satisfies $\sigma(i)<i$. We have $p(n, s, t, r)$ choices for $\sigma$ and the second term of the recurrence relation is obtained.

(c) If $\sigma$ has $n-s+1$ excedances, $t$ fixed points and $r$ cycles, then we can insert the entry $n+1$ just before one of the $n-s+1$ entries $\sigma(i)$ that satisfies $\sigma(i)>i$. We 
have $p(n, s-1, t, r)$ choices for $\sigma$ and the third term of the recurrence relation is obtained.

(d) If $\sigma$ has $n-s+1$ excedances, $t-1$ fixed points and $r-1$ cycles, then we can insert the entry $n+1$ at the end of $\sigma$ to form a new cycle $(n+1)$. We have $p(n, s-1, t-1, r-1)$ choices for $\sigma$ and the last term of the recurrence relation is obtained.

This completes the proof.

Equivalently, we can express Theorem 3.2 in terms of a differential equation.

Corollary 3.3. For $n \geq 0$,

$$
P_{n+1}(x, y, q)=(n x+y q) P_{n}(x, y, q)+x(1-x) \frac{\partial}{\partial x} P_{n}(x, y, q)+x(1-y) \frac{\partial}{\partial y} P_{n}(x, y, q) \text {. }
$$

Using (3.2), the polynomials $P_{n}(x, y, q)$ for $i=1, \ldots, 5$ are as follows:

$$
\begin{aligned}
P_{1}(x, y, q) & =q y, \\
P_{2}(x, y, q) & =q x+q^{2} y^{2}, \\
P_{3}(x, y, q) & =q\left(x+x^{2}\right)+3 x y q^{2}+q^{3} y^{3}, \\
P_{4}(x, y, q)= & q\left(x+4 x^{2}+x^{3}\right)+q^{2}\left(4 x y+4 x^{2} y+3 x^{2}\right)+6 x y^{2} q^{3}+q^{4} y^{4}, \\
P_{5}(x, y, q)= & q\left(x+11 x^{2}+11 x^{3}+x^{4}\right)+q^{2}\left(5 x y+10 x^{2}+20 x^{2} y+5 x^{3} y+10 x^{3}\right) \\
& \quad+q^{3}\left(10 x y^{2}+15 x^{2} y+10 x^{2} y^{2}\right)+10 x y^{3} q^{4}+q^{5} y^{5} .
\end{aligned}
$$

Let $D_{n, k}(x, q)$ be the coefficient of $y^{k}$ in $P_{n}(x, y, q)$. Note that the polynomial $D_{n, 0}(x, q)$ is the corresponding enumerative polynomial on $D_{n}$. Set $D_{n}(x, q)=$ $D_{n, 0}(x, q)$, that is, $D_{n}(x, q)=P_{n}(x, 0, q)$. Then

$$
\begin{aligned}
D_{n, k}(x, q) & =\sum_{\substack{\pi \in \mathcal{S}_{n} \\
\operatorname{fix}(\pi)=k}} x^{\operatorname{exc}(\pi)} q^{\operatorname{cyc}(\pi)} \\
& =\left(\begin{array}{l}
n \\
k
\end{array}\right) q^{k} \sum_{\sigma \in D_{n-k}} x^{\operatorname{exc}(\sigma)} q^{\operatorname{cyc}(\sigma)} \\
& =\left(\begin{array}{l}
n \\
k
\end{array}\right) q^{k} D_{n-k}(x, q) .
\end{aligned}
$$

Equating the coefficients in (3.2), we immediately derive

$$
D_{n+1}(x, q)=n x D_{n}(x, q)+x(1-x) \frac{\partial}{\partial x} D_{n}(x, q)+n q x D_{n-1}(x, q) .
$$

Let $\pi \in D_{n}$. Weighting $\pi$ by the monomial $x^{\operatorname{exc}(\pi)} q^{\operatorname{cyc}(\pi)}$, the bijective map from $\pi$ to its inverse $\pi^{-1}$ gives a monomial $x^{n-\operatorname{exc}(\pi)} q^{\operatorname{cyc}(\pi)}$. Hence $D_{n}(x, q)=x^{n} D_{n}\left(x^{-1}, q\right)$. The recurrence relation (3.3) enables us to show that the sequence of polynomials $\left\{D_{n}(x, q)\right\}_{n \geq 2}$ forms a Sturm sequence, where $q>0$. The proof turns out to be an application of the following lemma. 
Lemma 3.4 [8, Corollary 2.4]. Let $\left\{f_{n}(x)\right\}_{n \geq 0}$ be a sequence of polynomials with positive leading coefficient. Assume that

$$
f_{n+1}(x)=a_{n}(x) f_{n}(x)+b_{n}(x) f_{n}^{\prime}(x)+c_{n}(x) f_{n-1}(x) \quad \text { for } n \geq 1,
$$

where $a_{n}(x), b_{n}(x)$ and $c_{n}(x)$ are real polynomials such that

$$
\operatorname{deg} f_{n+1}(x)=\operatorname{deg} f_{n}(x)+1 .
$$

Suppose that for each $n$, the coefficients of $f_{n}(x)$ are nonnegative. If $b_{n}(x) \leq 0$ and $c_{n}(x) \leq 0$ whenever $f_{n}(x)=0$, then $\left\{f_{n}(x)\right\}_{n \geq 0}$ forms a Sturm sequence.

Thus we reach the following assertion.

Theorem 3.5. For $n \geq 2$, the polynomial $D_{n}(x, q)$ is symmetric and the sequence of polynomials $\left\{D_{n}(x, q)\right\}_{n \geq 2}$ forms a Sturm sequence, where $q>0$.

\section{Acknowledgement}

The author thanks the anonymous referee for his/her valuable suggestions.

\section{References}

[1] M. Bóna, Combinatorics of Permutations (Chapman \& Hall/CRC, Boca Raton, FL, 2004).

[2] P. Brändén, 'On linear transformations preserving the Pólya frequency property', Trans. Amer. Math. Soc. 358 (2006), 3697-3716.

[3] F. Brenti, 'A class of $q$-symmetric functions arising from plethysm', J. Combin. Theory Ser. A 91 (2000), 137-170.

[4] L. Comtet, Advanced Combinatorics (Reidel, Dordrecht, 1974).

[5] D. Foata and M. P. Schützenberger, Théorie géométrique des polynômes eulériens, Lecture Notes in Mathematics, 138 (Springer, New York, 1970).

[6] A. Kasraoui and J. Zeng, 'Euler-Mahonian statistics on ordered set partitions (II)', J. Combin. Theory Ser. A 116 (2009), 539-563.

[7] G. Ksavrelof and J. Zeng, 'Two involutions for signed excedance numbers', Sém. Lothar. Combin. 49 (2003), 8, Art. B49e.

[8] L. L. Liu and Y. Wang, 'A unified approach to polynomial sequences with only real zeros', $A d v$. in Appl. Math. 38 (2007), 542-560.

[9] S. M. Ma, 'A summation formula related to the $q$-Eulerian polynomials', Ars Combin. 94 (2010), 299-301.

[10] R. Mantaci and F. Rakotondrajao, "A permutation representation that knows what "Eulerian" means', Discrete Math. Theor. Comput. Sci. 4 (2001), 101-108.

[11] R. Mantaci and F. Rakotondrajao, 'Exceedingly deranging!', Adv. in Appl. Math. 30 (2003), 177-188.

[12] F. Rakotondrajao, 'Permutations by number of fixed points and anti-excedances', Afr. Mat., doi:10.1007/s13370-011-0025-y.

[13] R. P. Stanley, Enumerative Combinatorics, Vol. 1 (Cambridge University Press, Cambridge, 1997).

[14] R. P. Stanley, Enumerative Combinatorics, Vol. 2 (Cambridge University Press, Cambridge, 1999).

\section{SHI-MEI MA, Department of Information and Computing Science,}

Northeastern University at Qinhuangdao, Qinhuangdao, Hebei 066004, PR China e-mail: shimeima@yahoo.com.cn 